\section{RMD Open}

Rheumatic \&

Musculoskeletal Diseases

\title{
Rheumatology practice amidst the COVID-19 pandemic: a pragmatic view
}

\author{
Vasco C Romão (D), ${ }^{1,2}$ Inês Cordeiro, ${ }^{1,2}$ Carla Macieira, ${ }^{1}$ Filipa Oliveira-Ramos, ${ }^{1,2}$ \\ José Carlos Romeu, ${ }^{1}$ Carlos Miranda Rosa, ${ }^{1}$ Maria João Saavedra, ${ }^{1,2}$ \\ Fernando Saraiva, ${ }^{1,2}$ Elsa Vieira-Sousa, ${ }^{1,2}$ João Eurico Fonseca (D) ${ }^{1,2}$
}

To cite: Romão VC, Cordeiro I, Macieira C, et al. Rheumatology practice amidst the COVID-19 pandemic: a pragmatic view. RMD Open 2020;6:e001314. doi:10.1136/rmdopen-2020001314

Received 8 May 2020 Revised 7 June 2020

Accepted 8 June 2020
Check for updates

(C) Author(s) (or their employer(s)) 2020. Re-use permitted under CC BY-NC. No commercial re-use. See rights and permissions. Published by BMJ.

${ }^{1}$ Rheumatology Department, Hospital de Santa Maria, Centro Hospitalar Universitário Lisboa Norte, Lisbon Academic Medical Centre, Lisbon, Portugal ${ }^{2}$ Rheumatology Research Unit, Instituto de Medicina Molecular João Lobo Antunes, Faculdade de Medicina, Universidade de Lisboa, Lisbon, Portugal

Correspondence to João Eurico Fonseca, Rheumatology Department, Hospital de Santa Maria, Centro Hospitalar Universitário Lisboa Norte. Av Prof. Egas Moniz, 1649-035 Lisbon, Portugal; jec fonseca@gmail.com

\section{ABSTRACT}

The coronavirus disease 2019 (COVID-19) pandemic has come with many challenges for healthcare providers and patients alike. In addition to the direct burden it has placed on societies and health systems, it had a significant impact in the care of patients with chronic diseases, as healthcare resources were deployed to fight the crisis, and major travel and social restrictions were adopted. In the field of rheumatology, this has required notable efforts from departments and clinicians to adapt to the novel status quo and assure the follow-up of patients with rheumatic and musculoskeletal diseases. In the present viewpoint, we provide a practical approach to tackle this reality. Key measures include setting up preventive team management strategies, optimising communication with patients and reorganising patient care in all its dimensions. We then anticipate the nuances of rheumatology practice as restrictive measures are progressively lifted, while an effective vaccine is still pending. This includes the need to reimpose the same strategy as further waves unfold. Finally, we look ahead and address the lessons we can incorporate into postCOVID-19 rheumatology.

\section{INTRODUCTION}

In the last 3 months, societies worldwide have undergone major transformations to meet the challenges posed by the coronavirus disease 2019 (COVID-19) pandemic. ${ }^{1}{ }^{2}$ Particular strain was put on healthcare systems, which had to adapt to the dramatic rise in demand, by increasing response capacity and prioritising assistance to patients with COVID-19. This was instrumental in reducing direct mortality, alongside with the implementation of travel restriction and social isolation measures. ${ }^{3} 4$ However, it has also generated significant difficulties in the care of patients with other acute and chronic conditions, the extent of which is only now starting to be appreciated..$^{5-7}$ In particular, patients with rheumatic and musculoskeletal diseases (RMDs) are at risk of having their follow-up compromised, which might translate into future complications, due to uncontrolled disease and undetected serious adverse effects of ongoing treatments. ${ }^{8} 9$ To face this and respond to patient needs, rheumatology departments have to quickly respond to the changing scenario and readapt their organic structure. ${ }^{9}$ This requires noteworthy adaptive capacity from all stakeholders and active, creative planning to safeguard both patients and healthcare workers (HCWs).

It is thus important to reflect on the various aspects of reorganising rheumatology care to keep it running during the COVID-19 pandemic (figure 1). This extends from the initial suspension of non-urgent medical appointments, substituted as much as possible by teleconsultation, to the following months of progressive increase in selected face-to-face visits, while dealing with the persistence of severe acute respiratory syndrome coronavirus 2 (SARS-CoV-2) infection in the community. Herein, we detail a practical approach to this problem, mainly based on our experience at a large tertiary university hospital. First, we focus on general measures to manage and protect HCWs, and on the importance of clear communication with patients. Second, we propose a model to reorganise RMD patient care across all its dimensions. Third, we address the 'dayafter', projecting how can rheumatology practice and research adapt as restrictions are progressively waived, natural herd immunity slowly expands and widespread vaccination is still not available. Finally, we look ahead, focusing on the lessons learnt that we can incorporate into post-COVID-19 rheumatology. Due to the variability of the pandemic, resources and guidance across countries, the concepts here discussed are best seen as a framework aimed at clinicians and departments involved in the care of patients with RMDs, who can adapt it to best fit their specific reality. 


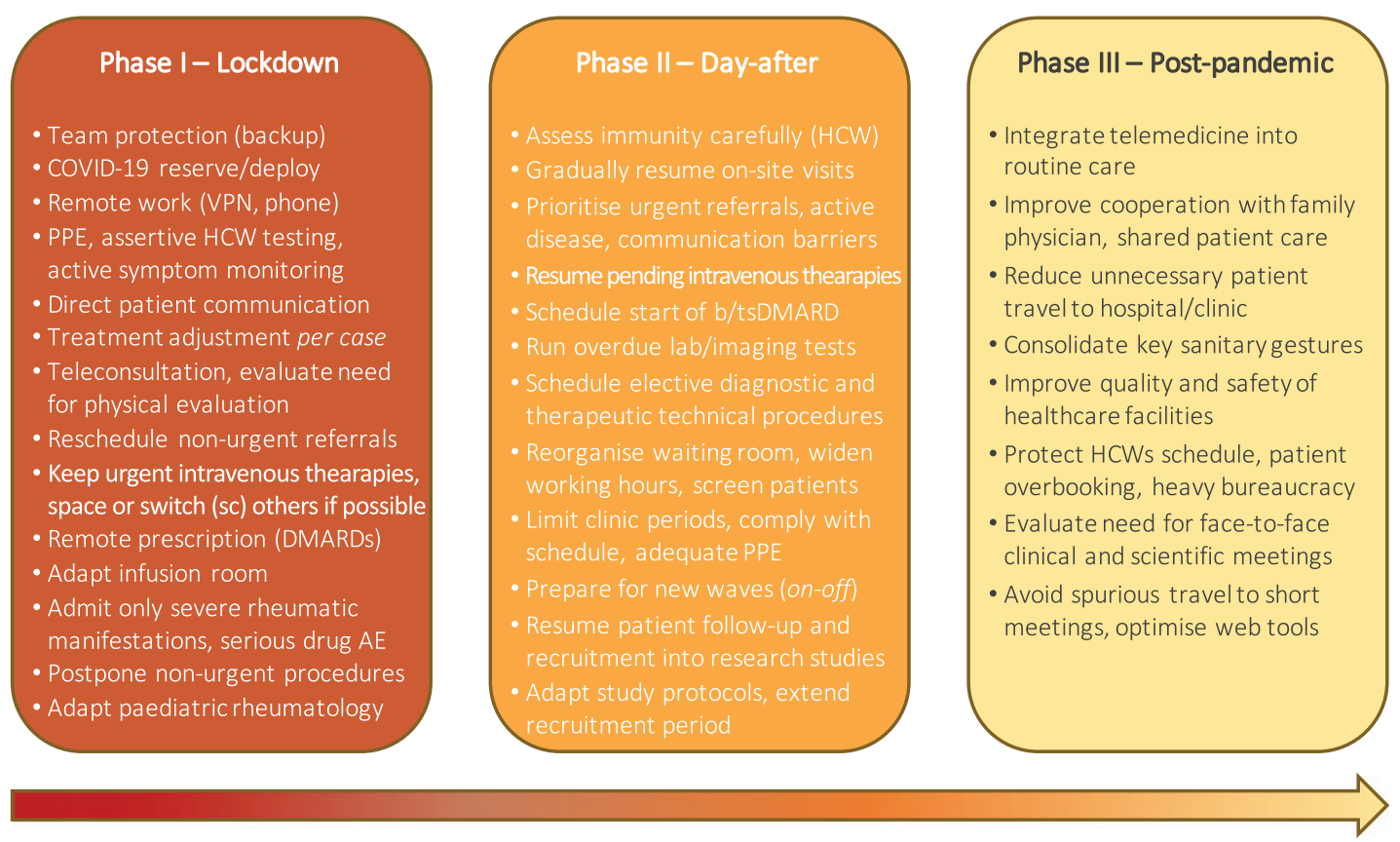

Figure 1 Reorganisation of rheumatology care during the COVID-19 pandemic. Sequential steps of organising rheumatology care throughout the coronavirus disease 19 (COVID-19) pandemic. Phase I addresses the initial response to the context of widespread community transmission of SARS-CoV-2, with major travel and social restrictions. Phase II refers to the adaptation taking place over the following months, as restrictions are slowly lifted and an effective vaccine is not yet available. Finally, phase III anticipates the prospect of post-COVID-19 rheumatology. AE, adverse effects; bDMARDs, biologic disease-modifying anti-rheumatic drugs; DMARDs, disease-modifying anti-rheumatic drugs; HCW, healthcare worker; IV, intravenous; PPE, personal protective equipment; SARS-CoV-2, severe acute respiratory syndrome coronavirus 2; sc, subcutaneous; tsDMARDs, targeted synthetic disease-modifying anti-rheumatic drugs; VPN, virtual private network.

\section{IMMEDIATE RESPONSE TO LOCKDOWN}

With a few exceptions, the initial response to the pandemic across the globe has generally included a more or less strict societal lockdown. This necessarily led to major adaptations in healthcare in general, and rheumatology practice in particular. While uncertain, future similarly restrictive lockdowns are a possibility, as potential surges in contagion ensue at the local, regional, or national level. In this event, implementation of comparable adjustments to patient care is expected to be required.

\section{General measures and team management}

Careful team management is a key element of the reorganisation response. Redistribution of HCWs in a rotative manner can be adopted, to assure the existence of backup elements at all times. This allows swift replacement of SARS-CoV-2-infected HCWs while promoting mental/ physical recovery in this stressful context. ${ }^{10}{ }^{11}$ Particular care may be employed with HCWs with clinical risk factors. Remote work (eg, administrative tasks, phone consultation, digital prescription) is a useful alternative whenever possible (eg, secure virtual private networks), with on-site support by a minimal number of staff. Depending on local/regional demands to reallocate resources to COVID-19-dedicated services, residents and younger specialists may be directly redeployed to these activities or remain as a COVID-19-specific reserve. It is important that this set-up remains fluid enough to naturally accommodate the dynamics of the pandemic and allow team redesign on a frequent basis.

Following local recommendations, universal surgical mask use and reinforcement of cornerstone protective gestures (eg, hand hygiene, social distancing) are fundamental in a hospital setting. ${ }^{12-15}$ This is particularly relevant among HCWs, as in-hospital transmission is now a recognised major issue. ${ }^{15-17}$ Other measures to be considered include frequent disinfection of common spaces and objects (eg, doorknobs, mouse, keyboard, desks), room ventilation improvement, and daily change of personal or medical clothing. ${ }^{12}$

Compliance with local occupational health guidance on symptom monitoring and testing of HCWs is needed. Testing strategies for SARS-CoV-2 by reverse transcription PCR (RT-PCR) vary among countries and clinical settings, depending on aspects such as type/duration of contact, local incidence, testing capacity and cost. Considering the transmission dynamics of SARS-CoV-2 (occurring at least 2-3 days before symptoms) ${ }^{18}$ the increasingly recognised importance of asymptomatic disease $(40-50 \%)^{19}$ and the hazardous consequences of widespread contagion among HCWs and patients, proactive and assertive screening measures are warranted in a healthcare setting. ${ }^{16}$ This includes testing symptomatic HCWs and quarantining direct close professional (if clinical workload allows) and personal 
contacts of confirmed cases. When feasible, extending testing to HCWs and patients exposed to viral-positive subjects would be preferable, to account for pre/asymptomatic carriers. ${ }^{20}$

Infected HCWs undergo self-isolation and distance monitoring for signs of severe disease by health authorities, occupational health and infectious disease specialists. ${ }^{12} 2122$ Additional follow-up by a department colleague might be helpful, can facilitate early detection of complications or arrangement of physical observation, and allows tracking of the team's working capacity. Return to work is advisable only after complete symptom resolution and negative RT-PCR testing. ${ }^{21-23}$ Due to delayed viral clearance (median time to negative test 20 days), ${ }^{24}{ }^{25}$ confirmation testing is worthwhile $3-7$ days after the disappearance of fever. ${ }^{21-23}$ Repeated testing is often necessary, ${ }^{24}{ }^{26}$ requiring teams to be prepared for frequent adjustments in work planning.

\section{Communicating with patients}

Assuring frank, accurate communication with patients is another crucial step. Patients with RMDs are likely to be anxious and need to feel supported by their assisting rheumatologists. As recently recognised in the European League Against Rheumatism (EULAR) provisional recommendations, ${ }^{20}$ there is currently no data to support that patients with RMDs, including those treated with disease-modifying anti-rheumatic drugs (DMARDs), have increased susceptibility to SARS-CoV-2 or a worse prognosis if infected. ${ }^{27-29}$ The reported higher overall risk of patients with systemic inflammatory RMDs to some infections (including viral infections) ${ }^{30-33}$ cannot, at the moment, be assumed for COVID-19. This message should be clearly conveyed to patients, who are advised to follow the same guidance as for the general population and patients without RMDs. ${ }^{20}$ Further, it is important to liaise with family practitioners, who also play an essential role in counselling and managing RMD patients.

It is worth mentioning, though, that patients with RMDs often have concomitant poor prognostic factors for COVID19 , such as advanced age (eg, those with osteoarthritis, pseudogout, giant cell arteritis), male sex (eg, those with gout), cardiovascular disease (eg, those with rheumatoid arthritis, systemic lupus erythematosus) or metabolic syndrome (eg, those with gout, psoriatic arthritis) ${ }^{244-37}$ Moreover, moderate-to-high-dose glucocorticoid usage, frequent in patients with connective tissue diseases (CTDs) and vasculitis, increases the risk of hospitalisation. ${ }^{28}$ In turn, tumour necrosis factor inhibitors have been associated with decreased odds of hospitalisation ${ }^{28}$ and, although evidence is overall weak, certain drugs have been used in specific stages of the infection, including tocilizumab, ${ }^{38} 39$ anakinra, ${ }^{40}$ baricitinib, ${ }^{41}$ immunoglobulin, ${ }^{42}$ chloroquine/ hydroxychloroquine, ${ }^{43}$ colchicine ${ }^{44}$ and glucocorticoids. ${ }^{45}$ Despite early suggestions, ${ }^{46}$ there is also no clear data to support a deleterious effect of nonsteroidal antiinflammatory drugs. ${ }^{47}$ It remains plausible, nonetheless, that some of these treatments can mask initial symptoms, delay diagnosis and confound the interpretation of a serious clinical complication of COVID-19.

Current recommendations advise patients with RMD to maintain their treatments. ${ }^{20} 4849$ If possible, increases in glucocorticoid dosage should be avoided.$^{48}$ As per regular follow-up, tapering of DMARDs/glucocorticoids may be considered in patients in persistent remission. ${ }^{50}{ }^{51}$ Besides general measures, patients may be counselled to stay at home whenever possible, use surgical masks when going out, stay physically and mentally active, eat healthily, rest frequently and cultivate sleep hygiene. ${ }^{52}$ Distance support over telephone/video consultation can be offered, along with a direct contact (secure phone/email) to the department/rheumatologist. ${ }^{20}$ It is important that patients are informed of suggestive COVID-19 symptoms and instructed to report them immediately to the department and national health authorities. If infected, glucocorticoids cannot be stopped abruptly and dosage modifications need to be carefully assessed. ${ }^{20}{ }^{48}$ Changes to ongoing DMARD therapy are best decided on a case-by-case basis, in a shared decision process. ${ }^{20}$ Close monitoring of the evolution of each case ensures patient's safety and facilitates enrolment in national/international registers. ${ }^{53} 54$

\section{Reorganising patient care}

The most challenging aspect of adapting to the pandemic is, undoubtedly, the reorganisation of patient care. This may vary depending on local policy, hospital/clinic context, volume of clinical activity and available facilities/ staff. The model herein proposed may be best adapted to local circumstances.

\section{Outpatient clinic}

In this context, remote telehealth consultations of patients with RMDs under previous follow-up are preferred. ${ }^{20} 48$ This aims at assessing clinical symptoms, evaluating results or need for blood tests, assuring prescriptions, providing psychological support, giving relevant information and scheduling a face-to-face appointment for a period of expected control of the pandemic. Patients with disease worsening, treatment complications or in whom physical evaluation is essential should discuss with their rheumatologist the risk-benefit of a face-to-face evaluation. ${ }^{20}{ }^{48}$ Likewise, the same attitude is preconised in urgent new outpatient referrals, including suspicious cases of inflammatory arthritides, CTDs and vasculitis. Other referrals should be postponed. Adequate support from, and collaboration with, family physicians is crucial in this stage, aiming at shared care of patients with RMDs.

\section{Management of targeted DMARDs and day care units}

Most patients treated with subcutaneous biologic (bDMARDs) and oral targeted synthetic DMARDs (tsDMARDs) can be safely monitored over telemedicine, reducing routine blood tests to the indispensable. Hospitals and national health systems might provide delivery of these drugs at home or at a local pharmacy, avoiding 
unnecessary travel to the hospital pharmacy. In the case of intravenous therapies, prioritisation might be necessary. Delaying treatment of patients with active CTDs and vasculitis with cyclophosphamide, rituximab, tocilizumab and belimumab may negatively impact the short-term outcome of these patients. On the contrary, spacing of infliximab can be a wise option in inflammatory arthritis patients with adequate disease control. ${ }^{48}$ For patients with stable disease activity and treated with intravenous tocilizumab, abatacept and belimumab, a discussion on the switch to subcutaneous formulations may be appropriate. Other possible strategies to reduce day care activity include carefully evaluating the spacing or need for intravenous immunoglobulin, iloprost (eg, consider phosphodiesterase-5 inhibitors) and zoledronate. Whenever reasonable, short-term delay of $b /$ tsDMARDs start may be considered, excluding patients with vital organthreatening disease. ${ }^{48}$

Specific measures may be necessary to assure the minimal functioning of day care facilities. Ideally, this would be in a non-COVID-19 area/building, with treatment schedules adapted to allow safe distancing $(>2 \mathrm{~m})$ at the infusion and waiting rooms, and patients coming in at a specific hour (when available seats are expected), wearing surgical masks. ${ }^{12}$ The use of personal protective equipment (PPE) adjusted to the possibility of contacting with asymptomatic/presymptomatic patients with COVID-19 by HCWs would be desirable, but may be limited by supply shortage. ${ }^{12-15}$ Frequent and between-patient disinfection of common surfaces is important to be observed. Screening of patients for COVID-19 symptoms and risk contacts prior to treatment (over telephone and on-site), coupled with temperature check and supervised hand sanitising, may contribute to increase safety. Systematic pre-treatment SARS-CoV-2 testing is likely not justified, but might be considered in specific cases (eg, potentially riskful contacts).

\section{Inpatient admissions}

Patient admissions should be reserved for acute, severe rheumatic manifestations and kept as short as possible. This will be mostly limited to severe manifestations of CTDs and vasculitis, and serious adverse effects of ongoing treatments. Depending on local practice, cases of infectious arthritis/spondylodiscitis may be admitted to rheumatology or orthopaedic wards. To account for presymptomatic/asymptomatic carriers and prevent inhospital outbreaks, it would be desirable that all admitted patients are tested for SARS-CoV-2. Urgent rheumatology support to other inpatients and to the emergency department may be guaranteed by a rotating rheumatologist in physical presence.

\section{Rheumatological procedures}

Diagnostic or therapeutic rheumatological technical procedures during lockdown are limited to patients that require undelayable physical evaluation. This mainly includes suspicions of infectious arthritis/tenosynovitis (synovial fluid aspiration) and giant cell arteritis (arterial ultrasound). As for other activities, protective measures ought to cover the possibility of contacting with asymptomatic/presymptomatic patients with COVID-19.

\section{Paediatric rheumatology}

The care of paediatric rheumatic patients can follow the same framework already described but does have a few singularities. There is a more frequent need for physical examination of new referrals and children with changes in symptom pattern, as reported by parents via phone consultation. Aiming at reducing circulating persons in healthcare facilities, it would be preferable if only one of the parents (or family member/legal guardian) is present in face-to-face appointments, infusion rooms or rheumatological procedures, following on-site evaluation of COVID-19 symptoms, temperature and hand sanitising. Surgical masks are required for all participants in the visits, except for children under 2 years of age. ${ }^{12-15} 55$ However, scaling PPE to account for presymptomatic/ asymptomatic COVID-19 cases may be considered in children difficult to examine, who often cry and cough, releasing respiratory droplets. Testing children prior to admission or to joint procedures performed under general anaesthesia or nitrous oxide sedation is desirable.

\section{DAY-AFTER: ADAPTING TO A LINGERING PANDEMIC}

Following the initial lockdown phase, we will have to deal with the COVID-19 threat for months to come. It is clearly unsustainable to maintain distance follow-up of patients with RMDs in the long run. Thus, adequate preparation and anticipation of the 'day-after' in rheumatology practice is needed, as restrictive measures are slowly lifted, but the menace of COVID-19 remains. If not larger, this will at least be as great an effort as the one detailed earlier. The long period it covers, along with the uncertainty associated, will test patients, physicians and other HCWs alike, to a degree we cannot yet clearly define.

\section{What about immunity?}

At present, most relevant questions regarding immunity developed to SARS-CoV-2 are largely unanswered and, thus, maximal caution is advised ${ }^{56}$ As serological assays become available, patients and HCWs can be tested to identify those who have developed an immunological response. ${ }^{57}$ This may include the identification of cases of asymptomatic infection or false-negative RT-PCR. ${ }^{57-59}$ While it is expected that most COVID-19 patients develop some kind of immune response, it has been shown that antibody titres can be of limited magnitude in up to $30 \%$ of cases and absent in up to $6 \% .{ }^{60}$ Also, it is not clear if existing IgG antibodies are always protective, at what titre, and for how long. ${ }^{56}{ }^{57}$ Nor is it known if the severity of infection influences the degree of immune response. ${ }^{2561}$ 
The impact of other factors such as age, sex, comorbidities and concomitant immunosuppressive therapy is also completely unknown and may play a role in patients with RMDs. ${ }^{56}$

For these reasons, careful interpretation of serology results needs to be ensured. Screening of HCWs in direct contact with suspected or confirmed patients with COVID-19 may be appropriate. However, a positive antiSARS-CoV-2 IgG does not justify, at the moment, changing the level of care or preventive measures by HCWs and patients. In particular, the immunological result to SARS-CoV-2 does not validate HCWs being assigned to different clinical tasks.

\section{Resuming on-site clinical care}

As of now and in upcoming months, on-site clinical care of patients with RMDs needs to be resumed. This will likely prove the greatest challenge of all, as it will still be a far different reality from what we were used to. In a phased, systematised way, patients will have to return to face-to-face consultations. Those with urgent referrals, active disease, serious manifestations or clear distance communication barriers may take priority. Initiation of $\mathrm{b} / \mathrm{tsDMARDs}$ can be programmed, and selected common technical procedures (eg, elective joint injections/aspirations) slowly scheduled. It is also important to guarantee that pending laboratory tests and other complementary exams are progressively performed. Finally, admission criteria for face-to-face consultations may be progressively widened. The nature and pace of these measures will vary, depending on local realities and pandemic evolution.

A few specific practical measures can be put forward. Waiting room capacity should allow safe intervals between patients and match the number of working consultation rooms. Extension of outpatient clinic working hours (eg, 8:00-20:00) may enable distribution of attending periods throughout the day. Patients are encouraged to come unaccompanied and at the precise time of the appointment. Physicians, in turn, should be compliant with set agendas and fixed consultation slots (eg, $30 \mathrm{~min}$ ). Further, it would be desirable that each clinician avoids extending clinic periods for long consecutive hours, to prevent fatigue and the consequent risk of neglecting safety procedures. Protective measures for patients and HCWs are likely to remain the same as those described earlier. In addition, testing for SARSCoV-2 may be justified prior to procedures such as minor salivary gland biopsies.

A pronounced bottleneck effect is to be expected. This results from several factors, including the delayed care of patients already under follow-up, the 2-months halt in new non-urgent referrals, as well as the slower pace of care as it is resumed. As such, patience, resilience and creativity are going to be in great demand for all of us in this period. In addition, the whole system may be tested further as potential additional contagion waves arise. Following the control of the first outburst and as restrictions are being softened, it is possible-and in fact expectable
- that a rise in cases and community transmission is observed. The extent of this upsurge is to be determined, but it may well grow enough to justify reinstitution of regional or national lockdown and, as a consequence, of the clinical measures described earlier. Such an on-andoff approach is likely to place too heavy a toll on the health system, HCWs and patients, the consequences of which we cannot even foresee.

\section{Keeping research, training and education going}

While most attention is naturally placed in minoring the clinical consequences in the care of patients with RMDs, one cannot forget the importance of maintaining clinical and translation research activities, and assuring undergraduate and postgraduate medical training. ${ }^{62} 64$

Clinical trials and observational studies are key to advancing the knowledge of all aspects of RMDs. Moreover, there has been tremendous investment in effort and resources that cannot be wasted. It is thus vital that clinicians, patients and researchers work together to ensure patient retention and slowly resume patient recruitment and the set-up of new studies. ${ }^{62}{ }^{65}$ Innovative trials, especially phase I/II, are likely to encounter additional reluctance from patients, who might be wary of potential side effects and increased vulnerability to COVID-19. Frequent visits and travel to the hospital may be yet another setback for patient inclusion. In this regard, investigators play an essential role in keeping patient safety as the utmost priority, while minimising loss to follow-up and missing data. Specific on-site measures similar to the described earlier may be necessary as part of a COVID19 action plan. Academic and industry sponsors should consider minor protocol amendments, such as allowing remote assessments, limiting non-essential visits, incorporating regular SARS-CoV-2 testing during trials and extending recruitment periods. ${ }^{62}{ }^{65}$ Collaborative work involving all parties is needed more than ever to keep research ongoing.

Preclinical and clinical medical training and education have also been severely disrupted by the pandemic. Globally, medical schools were forced to close and started online teaching on short-notice, aiming to protect both students and patients, but greatly affecting clinical practice training. ${ }^{63}$ Likewise, speciality training was halted for 2-3 months, as routine clinical work stopped and trainees were enrolled in COVID-19-centred teams, while still collaborating in telemedicine activities. ${ }^{64}$ As patient care is progressively resumed, trainees may be incorporated in previous rotations, developing supervised clinical work in outpatient, inpatient, day care and rheumatological procedures settings. Speciality training programmes may need to be extended to compensate for the break in specific formation. ${ }^{64}$ Accompanying consultants in patient visits may no longer be wise, to avoid overcrowding. This will prove particularly challenging for undergraduate rheumatology training, requiring low student-to -patient ratios. It may require profound reorganisation of medical curricula, extending clinical rotations for longer 
periods, to allow for better student distribution throughout the year. ${ }^{63}$

\section{LOOKING AHEAD: POST-COVID-19 RHEUMATOLOGY}

As the post-pandemic era is still little more than a mirage, society in general and healthcare in particular should not shy away from taking valuable lessons from these unprecedented events. The post-COVID-19 period will hardly be the same as it was up to early 2020, and the field of RMDs is no exception. It is our responsibility to quickly begin thinking about it and incorporate strategies that will slowly change the way we currently practise rheumatology.

It is important to first acknowledge the potential of telemedicine, including telephone and video consultations. ${ }^{66}$ At least a part of routine follow-up visits may easily be adapted to this form, saving unnecessary patient travel, while still assuring care. To assure this, optimal communication and cooperation between rheumatologists and family medicine physicians is of paramount importance. ${ }^{67}$ We will have the unique opportunity to consolidate shared care as standard practice for patients with RMDs. Simply allowing patients to do blood tests or imaging exams in ambulatory clinics may greatly reduce patient flow and accumulation in common hospital areas. Simultaneously, reinforcing patient and HCW education in fundamental preventive gestures, such as frequent hand washing, social distancing and symptom monitoring will aid reducing transmission of this and other infectious diseases. Assurance of safe, large, adequate healthcare facilities for outpatient, inpatient and day care is important to remain the norm after the pandemic fades. Clinical and scientific regular meetings can be reassessed for the need of physical presence and optimised to reduce wasteful time. In particular, classical ward rounds may have to be reassessed for their learning potential and progressively abandoned to comply with precautionary measures. Protection of HCWs in terms of long hours, agenda burden, patient overbooking and bureaucratic tasks needs to be observed. Alternatives are required to physician and researcher travel to short meetings, taking advantage of digital web conferencing tools.

\section{CONCLUSIONS}

The COVID-19 pandemic has brought tremendous challenges upon rheumatologists and patients with RMDs. Together, we have so far been able to adapt to such exceptional circumstances and respond in a fast, effective fashion to secure immediate clinical care. As lockdowns start to be removed, we will be faced with novel demands, that will test us and our institutions for a long period. Hopefully, we can minimise the impact of this common opponent, learn and grow from the struggle. The future in rheumatology will, undoubtedly, be influenced by this event and we all have a role to play in designing it, starting from today.
Contributors All authors have contributed to study conception and design. VCR and JEF drafted the manuscript, which was critically reviewed by all authors. All authors have read and approved the final version of the manuscript.

Funding The authors have not declared a specific grant for this research from any funding agency in the public, commercial or not-for-profit sectors.

Competing interests None declared.

Patient consent for publication Not required.

Provenance and peer review Not commissioned; externally peer reviewed.

Open access This is an open access article distributed in accordance with the Creative Commons Attribution Non Commercial (CC BY-NC 4.0) license, which permits others to distribute, remix, adapt, build upon this work non-commercially, and license their derivative works on different terms, provided the original work is properly cited, appropriate credit is given, any changes made indicated, and the use is non-commercial. See: http://creativecommons.org/licenses/by-nc/4.0/.

\section{ORCID iDs}

Vasco C Romão http://orcid.org/0000-0002-5603-9436

João Eurico Fonseca http://orcid.org/0000-0003-1432-3671

\section{REFERENCES}

1 World Health Organization. WHO coronavirus disease (COVID-2019) situation report - 101 [Internet]. Available https://www.who.int/docs/ default-source/coronaviruse/situation-reports/20200430-sitrep-101covid-19.pdf?sfvrsn=2ba4e093 2 (accessed 2020 Apr 30)

2 Sohrabi C, Alsafi Z, O'Neill N, et al. World Health Organization declares global emergency: a review of the 2019 novel coronavirus (COVID-19). Int J Surg 2020;76:71-6.

3 Walker PG, Whittaker C, Watson O, et al. The global impact of COVID-19 and strategies for mitigation and suppression. Imp Coll COVID-19 Response Team 2020;2020:1-19.

4 Zhang J, Litvinova M, Liang Y, et al. Changes in contact patterns shape the dynamics of the COVID-19 outbreak in China. Science (80-) 2020;8001:eabb8001.

5 Saini KS, de Las Heras B, de Castro J, et al. Effect of the COVID-19 pandemic on cancer treatment and research. Lancet Haematol 2020;2019:2019-21.

6 Tam CCF, Cheung KS, Lam S, et al. Impact of coronavirus disease 2019 (COVID-19) outbreak on ST-segment-elevation myocardial infarction care in Hong Kong, China. Circ Cardiovasc Qual Outcomes 2020;2019:(April):2019-21.

7 American Cancer Society Cancer Action Network. COVID-19 pandemic impact on cancer patients and survivors survey: findings summary [internet]. Am. Cancer Soc. Cancer Action Netw. 2020; Available https://www.fightcancer.org/policy-resources/covid-19pandemic-impact-cancer-patients-and-survivors-survey-findingssummary (accessed 2020 Apr 30)

8 Mclnnes IB. COVID-19 and rheumatology: first steps towards a different future? Ann Rheum Dis 2020;79:551-2.

9 Lewandowski LB, Hsieh E. Global rheumatology in the time of COVID-19. Lancet Rheumatol 2020;9913:2019-20.

10 American Medical Association. Caring for our caregivers during COVID-19. Am Med Assoc 2020.

11 Shanafelt T, Ripp J, Understanding TM. Addressing sources of anxiety among health care professionals during the COVID-19 pandemic. J Am Med Assoc 2020;2019:2019-20.

12 European Centre for Disease Prevention and Control. Infection prevention and control and preparedness for COVID-19 in healthcare settings: second update. 31 March 2020. [Internet]. 2020; Available https://www.ecdc.europa.eu/sites/default/files/documents/ Infection-prevention-control-for-the-care-of-patients-with-2019nCoV-healthcare-settings_update-31-March-2020.pdf (accessed 2020 Apr 28).

13 Klompas M, Morris CA, Sinclair J, et al. Universal masking in hospitals in the COVID-19 era. N Engl J Med 2020;382:e63.

14 Chu DK, Akl EA, Duda S, et al. Physical distancing, face masks, and eye protection to prevent person-to-person transmission of SARS-CoV-2 and COVID-19: a systematic review and meta-analysis. Lancet 2020;6736:1-15.

15 Heinzerling A, Stuckey MJ, Scheuer T, et al. Transmission of COVID-19 to health care personnel during exposures to a hospitalized patient Solano County, California, February 2020. MMWR Morb Mortal Wkly Rep 2020;69:472-6.

16 Black JRM, Bailey C, Przewrocka J, et al. COVID-19: The case for health-care worker screening to prevent hospital transmission. Lancet 2020;395:1418-20. 
17 Wang D, Hu B, Hu C, et al. Clinical characteristics of 138 hospitalized patients with 2019 novel coronavirus: infected pneumonia in Wuhan, China. JAMA - J Am Med Assoc 2020;323:1061-9.

$18 \mathrm{He}$ X, Lau EHY, Wu P, et al. Temporal dynamics in viral shedding and transmissibility of COVID-19. Nat Med 2020;26:672-5.

19 Oran DP, Topol EJ. Prevalence of asymptomatic SARS-CoV-2 infection. Ann Intern Med 2020:M20-3012.

20 Landewé RB, Machado PM, Kroon F, et al. EULAR provisional recommendations for the management of rheumatic and musculoskeletal diseases in the context of SARS-CoV-2. Ann Rheum Dis 2020; annrheumdis-2020-217877.

21 European Centre for Disease Prevention and Control. Guidance for discharge and ending isolation in the context of widespread community transmission of COVID-19, 8 April 2020. [Internet]. 2020; Available https://www.ecdc.europa.eu/en/publications-data/covid19-guidance-discharge-and-ending-isolation (accessed 29 Apr 2020)

22 World Health Organization. Clinical management of severe acute respiratory infection when COVID-19 is suspected 2020. (13 Mar 2020). [Internet]. 2020; Available https://apps.who.int/iris/handle/10665/ 331446 (accessed 29 Apr 2020)

23 Centers for Disease Control and Prevention. Criteria for return to work for healthcare personnel with suspected or confirmed COVID-19 (Interim Guidance). [Internet]. 2020; Available https://www.cdc.gov/coronavirus/ 2019-ncov/hcp/return-to-work.html (accessed 30 Apr 2020)

24 Zhou F, Yu T, Du R, et al. Clinical course and risk factors for mortality of adult inpatients with COVID-19 in Wuhan, China: a retrospective cohort study. Lancet 2020;395:1054-62.

25 Wölfel R, Corman VM, Guggemos W, et al. Virological assessment of hospitalized patients with COVID-2019. Nature 2020.

26 Lan L, Xu D, Ye G, et al. Positive RT-PCR test results in patients recovered from COVID-19. JAMA 2020;323:1502.

27 Haberman R, Axelrad J, Chen A, et al. COVID-19 in immune-mediated inflammatory diseases: case series from New York. N Engl J Med 2020; NEJMc2009567.

28 Gianfrancesco M, Hyrich KL, Al-Adely S, et al. Characteristics associated with hospitalisation for COVID-19 in people with rheumatic disease: data from the COVID-19 global rheumatology alliance physician-reported registry. Ann Rheum Dis 2020;1-8.

29 D'Silva KM, Serling-Boyd N, Wallwork R, et al. Clinical characteristics and outcomes of patients with coronavirus disease 2019 (COVID-19) and rheumatic disease: a comparative cohort study from a US "hot spot". Ann Rheum Dis 2020;1-7.

30 Singh JA, Cameron C, Noorbaloochi S, et al. Risk of serious infection in biological treatment of patients with rheumatoid arthritis: a systematic review and meta-analysis. Lancet 2015;386:258-65.

31 Atzeni F, Bendtzen K, Bobbio-Pallavicini F, et al. Infections and treatment of patients with rheumatic diseases. Clin Exp Rheumatol 2008;26:S67-73-.

32 Khasnis AA, Schoen RT, Calabrese LH. Emerging viral infections in rheumatic diseases. Semin Arthritis Rheum 2011;41:236-46.

$33 \mathrm{Hsu} \mathrm{C}-\mathrm{Y}, \mathrm{Ko} \mathrm{C}-\mathrm{H}$, Wang J-L, et al. Comparing the burdens of opportunistic infections among patients with systemic rheumatic diseases: a nationally representative cohort study. Arthritis Res Ther 2019;21:1-10.

34 Yang J, Zheng Y, Gou X, et al. Prevalence of comorbidities and its effects in patients infected with SARS-CoV-2: a systematic review and meta-analysis. Int J Infect Dis 2020;94:91-5.

35 Chen T, Wu D, Chen $\mathrm{H}$, et al. Clinical characteristics of 113 deceased patients with coronavirus disease 2019: retrospective study. BMJ 2020;368:m1091.

36 Mehra MR, Desai SS, Kuy S, et al. Cardiovascular disease, drug therapy, and mortality in COVID-19. N Engl J Med 2020; NEJMoa2007621.

37 Nurmohamed MT, Heslinga M, Kitas GD. Cardiovascular comorbidity in rheumatic diseases. Nat Rev Rheumatol 2020;323:693-704.

38 Tocilizumab improves significantly clinical outcomes of patients with moderate or severe COVID-19 pneumonia. [Internet]. 2020. Available https://www.aphp.fr/contenu/tocilizumab-improves-significantlyclinical-outcomes-patients-moderate-or-severe-covid-19 (accessed 28 Apr 2020)

$39 \mathrm{Xu}$ X, Han M, Li T, et al. Effective treatment of severe COVID-19 patients with tocilizumab. Proc Natl Acad Sci 2020;202005615

40 Mehta P, McAuley DF, Brown M, et al. COVID-19: consider cytokine storm syndromes and immunosuppression. Lancet 2020;395:1033-4.

41 Cantini F, Niccoli L, Matarrese D, et al. Baricitinib therapy in COVID-19: a pilot study on safety and clinical impact. $J$ Infect 2020;2020:(16 April 2020).
42 Xie Y, Cao S, Dong H, et al. Effect of regular intravenous immunoglobulin therapy on prognosis of severe pneumonia in patients with COVID-19. J Infect 2020. (accessed 26 Mar 2020)

43 AV H, Roman YM, Pasupuleti V, et al. Hydroxychloroquine or chloroquine for treatment or prophylaxis of COVID-19: a living systematic review. Ann Intern Med 2020.

44 Deftereos S, Giannopoulos G, Vrachatis DA, et al. Colchicine as a potent anti-inflammatory treatment in COVID-19: can we teach an old dog new tricks? Eur Hear J Cardiovasc Pharmacother 2020;12462.

45 Villar J, Confalonieri M, Pastores SM, et al. Rationale for prolonged corticosteroid treatment in the acute respiratory distress syndrome caused by coronavirus disease 2019. Crit Care Explor 2020;2:e0111.

46 Fang L, Karakiulakis G, Roth M. Are patients with hypertension and diabetes mellitus at increased risk for COVID-19 infection? Lancet Respir Med 2020;8:e21.

47 World Health Organization. The use of non-steroidal anti-inflammatory drugs (NSAIDs) in patients with COVID-19: scientific brief. [Internet]. 2020; https://apps.who.int/iris/bitstream/handle/10665/331796/ WHO-2019-nCoV-Sci Brief-NS (accessed 27 Apr 2020)

48 Mikuls TR, Johnson SR, Fraenkel L, et al. American College of Rheumatology guidance for the management of rheumatic disease in adult patients during the COVID-19 pandemic: version 1. Arthritis Rheumatol 2020;1-11.

49 Matucci-Cerinic M, Bruni C, Allanore Y, et al. Systemic sclerosis and the COVID-19 pandemic: world scleroderma foundation preliminary advice for patient management. Ann Rheum Dis 2020;1-3.

50 Schett G, Emery P, Tanaka Y, et al. Tapering biologic and conventional DMARD therapy in rheumatoid arthritis: current evidence and future directions. Ann Rheum Dis 2016;75:1428-37.

51 Ziade NR, Baraliakos X. Tapering bDMARDs in axial SpA: what is the current evidence? Nat Rev Rheumatol 2019;15:322-4.

52 Pinto AJ, Dunstan DW, Owen N, et al. Combating physical inactivity during the COVID-19 pandemic. Nat Rev Rheumatol 2020;19-20.

53 Canhao H, Faustino A, Martins F, et al. Reuma.pt - the rheumatic diseases Portuguese register. Acta Reumatol Port 2011;36:45-56.

54 Robinson PC, Yazdany J. The COVID-19 global rheumatology alliance: collecting data in a pandemic. Nat Rev Rheumatol 2020.

55 CDC. Centers for disease control and prevention. Use of cloth face coverings to help slow the spread of COVID-19 [Internet]. 2020. Available https://www.cdc.gov/coronavirus/2019-ncov/preventgetting-sick/diy-cloth-face-coverings.html (accessed 29 Apr 2020)

56 Kellam P, Barclay W. The dynamics of humoral immune responses following SARS-CoV-2 infection and the potential for reinfection. J Gen Virol 2020

57 Cheng MP, Yansouni CP, Basta NE, et al. Serodiagnostics for severe acute respiratory syndrome: related coronavirus-2. Ann Intern Med 2020;0:M20-2854-

58 Long Q, Liu B-Z, Deng H-J, et al. Antibody responses to SARS-CoV-2 in patients with COVID-19. Nat Med 2020.

59 Kontou PI, Braliou GG, Dimou NL, et al. Antibody tests in detecting SARS-CoV-2 infection: a meta-analysis. Diagnostics 2020;10:319.

60 Wu F, Wang A, Liu M, et al. Neutralizing antibody responses to SARS-CoV-2 in a COVID-19 recovered patient cohort and their implications. medRxiv 2020.

61 To KK-W, Tsang OT-Y, Leung W-S, et al. Temporal profiles of viral load in posterior oropharyngeal saliva samples and serum antibody responses during infection by SARS-CoV-2: an observational cohort study. Lancet Infect Dis 2020;20:565-74.

62 Gewin V. Safely conducting essential research in the face of COVID-19. Nature 2020;580:549-50.

63 Rose S. Medical student education in the time of COVID-19. JAMA 2020;323:2131-2.

64 Fitzpatick M, Roycroft M, Hynes G Towards a 'new normal' for physician training following COVID-19 [Internet]. R. Coll. Physicians. 2020. Available https://www.rcplondon.ac.uk/news/towards-newnormal-physician-training-following-covid-19 (accessed 29 May 2020)

65 EMA (European Medicines Agency). Guidance on the management of clinical trials during the COVID-19 (Coronavirus) pandemic - version 3. 2020. V3 (accessed 28 Apr 2020).

66 Perniola S, Alivernini S, Varriano V, et al. Telemedicine will not keep us apart in COVID-19 pandemic. Ann Rheum Dis 2020;0:annrheumdis2020-218022.

67 Lim AYN, Tan C-S, Low BPL, et al. Integrating rheumatology care in the community: can shared care work? Int J Integr Care 2015;15:1-12. 